\title{
Population pharmacokinetics of sirolimus in pediatric patients with kaposiform hemangioendothelioma: A retrospective study
}

\author{
DONGDONG WANG ${ }^{1 *}$, XIAO $\mathrm{CHEN}^{1,2^{*}}$ and ZHIPING $\mathrm{LI}^{1}$ \\ ${ }^{1}$ Department of Pharmacy, Children's Hospital of Fudan University, Shanghai 201102; \\ ${ }^{2}$ Department of Pharmacy, The People's Hospital of Jiangyin, Jiangyin, Jiangsu 214400, P.R. China
}

Received October 16, 2018; Accepted June 11, 2019

DOI: $10.3892 / \mathrm{ol} .2019 .10562$

\begin{abstract}
Numerous studies have established population pharmacokinetics (PPK) models of sirolimus in various populations. However, a PPK model of sirolimus in Chinese patients with pediatric kaposiform hemangioendothelioma (PKHE) has yet to be established; therefore, this was the purpose of the present study. The present study was a retrospective analysis that utilized the trough concentration data obtained from traditional therapeutic drug monitoring-based dose adjustments. A total of 17 Chinese patients with PKHE from a real-world study were characterized by non-linear mixed-effects modeling. The impact of demographic features, biological characteristics and concomitant medications was assessed. The developed final model was evaluated via bootstrap and a prediction-corrected visual predictive check. A one-compartment model with first-order absorption and elimination was used for modeling of data for PKHE. The typical values of apparent oral clearance $(\mathrm{CL} / \mathrm{F})$ and apparent volume of distribution $(\mathrm{V} / \mathrm{F})$ in the final model were $3.19 \mathrm{l} / \mathrm{h}$ and 165 liters, respectively. Age, alanine transaminase levels and sex were included as significant covariates for $\mathrm{CL} / \mathrm{F}$, while the duration of treatment with sirolimus was a significant covariate for $\mathrm{V} / \mathrm{F}$. In conclusion, the present study developed and validated the first sirolimus PPK model for Chinese patients with PKHE.
\end{abstract}

\section{Introduction}

Kaposiform hemangioendothelioma (KHE) is a rare vascular tumor type with a prevalence of 0.9/100,000 children between 1991 and 2009 in the USA (1). It affects the head/neck region, the extremities and the trunk, and even occurs in the

Correspondence to: Professor Zhiping Li, Department of Pharmacy, Children's Hospital of Fudan University, 399 Wanyuan Road, Shanghai 201102, P.R. China

E-mail: zplifudan@126.com

*Contributed equally

Key words: pediatric, kaposiform hemangioendothelioma, population pharmacokinetics, sirolimus, real-world study retroperitoneal or thoracic cavity, with invasion of the skin, subcutaneous fat and muscle (2). KHE triggers pain and causes deformity. Additionally, this local aggressive lesion may cause platelet trapping, leading to profound thrombocytopenia, an enlarged lesion and a consumptive coagulopathy with significant hypofibrinogenemia, in a process termed Kasabach-Merritt phenomenon (KMP) (3). While KHE is a benign lesion type, KMP poses a significant threat to the life of affected patients, and the mortality rate was as high as $12-30 \%$ between 1991 and 2009 due to various factors, including local invasion, hemodynamic instability and compression of vital structures $(4,5)$. Therefore, it is necessary to develop treatments for KHE.

Various studies have described that sirolimus, an inhibitor of mTOR, is a treatment option for KHE (6-10). However, sirolimus exhibits considerable inter- and intra-individual variabilities in its pharmacokinetics (PK), with a poor association between the drug dosage and blood concentrations, which makes it difficult to specify an optimal dose $(11,12)$.

Population pharmacokinetics (PPK) collects PK information from sparse data on patients $(13,14)$. PPK analysis is able to distinguish inter-individual variability and residual unexplained confounders (15). Therefore, PPK has a greater statistical power for verifying the effect of multiple factors on the PK of sirolimus compared with that of traditional PK analysis (16), making it possible to define an optimal dose schedule.

However, the sirolimus PPK model in Chinese patients with pediatric KHE (PKHE), to the best of our knowledge, remains undefined. The objective of the present study was to establish a PPK model for sirolimus in a Chinese PKHE population, and to identify factors that account for the PK variability, in order to optimize individualized therapy.

\section{Materials and methods}

Patients and data collection. Data for a total of 17 Chinese patients with PKHE (11 males and 6 females; age, 0.2-6 years; mean age, $1.21 \pm 1.2$ years) who presented at the Children's Hospital of Fudan University between January 2016 and April 2018, were retrospectively analyzed. Data on the drug concentrations and relevant clinical information were obtained from therapeutic drug monitoring (TDM) records and medical records, respectively. The present study was approved by the 
Research Ethics Committee of Children's Hospital of Fudan University.

Information collected from the medical records included sex, age, weight, duration of treatment with sirolimus (DTT), daily dose of sirolimus (DDS), alanine transaminase (ALT), aspartate transaminase (AST), creatinine $(\mathrm{Cr})$, urea (UR), hematocrit (HCT), hemoglobin (HGB), mean corpuscular hemoglobin $(\mathrm{MCH})$, mean corpuscular hemoglobin concentration (MCHC) and concomitant treatment with other drugs (sulfamethoxazole, methylprednisolone, digoxin, ursodeoxycholic, cimetidine, omeprazole, fluconazole, cefoperazone/sulbactam and voriconazole).

Drug administration. Sirolimus was administered orally on a continuous dosing schedule at a starting dose of $0.8 \mathrm{mg} / \mathrm{m}^{2}$ (where $\mathrm{m}^{2}$ represents body surface area) per dose twice daily (10), and all of the blood concentrations were collected prior to the next administration. Peripheral blood was collected by the blood collection tube with EDTA, which could be stored at $18-25^{\circ} \mathrm{C}$ for $24 \mathrm{~h}$ (according to the instructions). The drug concentration was measured two times per week or more frequently if required (e.g. in the case of suspicion of intolerance or adverse events) using an automatic biochemical analyzer (Viva-E; Siemens AG). The trough levels were maintained between 10 and $15 \mathrm{ng} / \mathrm{ml}(10)$.

Analytical method. The blood concentration of sirolimus was measured using an Emit 2000 Sirolimus Assay (Siemens Healthcare Diagnostics, Inc.). Key assay performance parameters were as follows: Lower limit of quantitation, $3.5 \mathrm{ng} / \mathrm{ml}$; range of linear response, 3.5-30 ng/ml; values of inter-assay variability [coefficient of variation $(\mathrm{CV} \%)$ ], $<4.0 \%$; and values of intra-assay CV $(\%),<6.2 \%$.

PPK modeling. Data were analyzed using the non-linear mixed-effects model (NONMEM) computer program (version VII; ICON Development Solutions, LLC). The first-order conditional estimation method with interaction option was used to estimate PK parameters and their variability. A one-compartment model with first-order absorption was applied to depict the absorption phase, since all sirolimus concentrations in the present study were trough concentrations. It was not possible to estimate the bioavailability $(\mathrm{F})$ and absorption rate constant with a lag time, since the concentration data were insufficient. Therefore, the PK parameters comprised apparent oral clearance $(\mathrm{CL} / \mathrm{F})$ and apparent volume of distribution $(\mathrm{V} / \mathrm{F})$. The absorption rate constant $\mathrm{K}_{\mathrm{a}}$ of the model was fixed to $0.485 \mathrm{~h}^{-1}$ based on a previous study (17).

Random-effect model. The inter-individual variability of the PK parameters was explored using an exponential error model (17). The value of a parameter in an individual $\left(P_{j}\right)$ was a function of the parameter value in the typical individual $[\mathrm{TV}(P)]$ and an individual deviation represented by $\eta_{i}$. It was assumed that $\eta_{i}$ was symmetrically distributed in the population, and the variance of zero-mean random variables was estimated as part of the model estimation from equation 1 :

$$
P_{i}=T V(P) \times \exp \left(\eta_{i}\right)
$$

Various statistical models (equations 2-4) describing the random residual variability were considered:

$$
\begin{aligned}
& Y=I P R E D+\varepsilon \\
& Y=I P R E D \times \exp (\varepsilon) \\
& Y=I P R E D \times \exp \left(\varepsilon_{1}\right)+\varepsilon_{2}
\end{aligned}
$$

where $Y$ represents the observation, IPRED is the individual predicted concentration and $\varepsilon_{n}$ is symmetrically distributed, for zero-mean random variables with variance terms that are estimated as part of the population model fitting process. Based on the minimum objective function value (OFV), equation (3) was finally selected to calculate the residual variability.

Covariate model. In order to explain the variability of PK parameters, the associations among covariates and all the PK parameters were investigated. The potential covariates included sex, age, weight, DTT, DDS, ALT, AST, Cr, UR, HCT, HGB, MCH, MCHC and concomitant medications. The covariate model was set up in a stepwise manner (15). To compare hierarchical models, a likelihood ratio test was adopted. The change in OFV caused by the inclusion of a covariate is proportional to twice the negative log likelihood of the data and approximates a $\chi^{2}$ distribution. In the univariate analysis, a decrease in the OFV by $>3.84(\mathrm{P}<0.05$; degrees of freedom $=1$ ) was used as a criterion for inclusion of the covariate in the base model (15). The significant covariate-parameter associations were reserved in the model. When a full regression model was built, the model was further tested by dropping one covariate from each parameter at a time to acquire the final model. An increase in the OFV by $>6.64(\mathrm{P}<0.01$; degrees of freedom $=1)$ was used as the criterion for retaining significant covariate-parameter associations in the model (15).

Model evaluation. An internal evaluation method using bootstrap was used to assess the stability and reliability of the final parameter estimates (15). Bootstrap was produced by repeated random sampling with replacement from the original data. This procedure was performed with the software package Wings for NONMEM (version VII; ICON Development Solutions, LLC), and repeated 1,000 times with different random draws. The medians and 2.5 th- 97.5 th percentiles of the bootstrap results were compared with the final PK parameter estimates (15). The goodness-of-fit plots were generated by $\mathrm{R}$ (version 3.4.2, http://www.r-project.org). The distribution of weighted residuals for the final model was assessed using histograms and Q-Q plots. The predictive performance of the final model was also assessed by a prediction-corrected visual predictive check Xpose (18) package (version 4.6.1) and $\mathrm{R}$ (version 3.4.2)].

\section{Results}

Data collection. The data obtained from 17 Chinese pediatric patients with KHE were included in the present analysis. A total of 133 blood concentration values were available for analysis. The patient characteristics and drug combinations are summarized in Tables I and II. 
Table I. Clinicopathological and demographic data of patients.

\begin{tabular}{lcc}
\hline Characteristic & Mean \pm SD & Median (range) \\
\hline Gender (male/female) & $11 / 6$ & $\mathrm{n} / \mathrm{a}$ \\
Age (years) & $1.21 \pm 1.2$ & $0.8(0.2-6)$ \\
Weight $(\mathrm{kg})$ & $7.99 \pm 3.04$ & $7(3.6-18)$ \\
Duration of treatment with sirolimus (days) & $154.2 \pm 149$ & $117(7-749)$ \\
Daily dose of sirolimus (mg) & $0.63 \pm 0.36$ & $0.5(0.16-1.5)$ \\
Alanine transaminase (IU/l) & $24.37 \pm 19.95$ & $21(2-120)$ \\
Aspartate transaminase $(\mathrm{IU} / \mathrm{l})$ & $37.26 \pm 23.52$ & $35(12-165)$ \\
Creatinine $(\mu$ mol/l) & $18.68 \pm 3.76$ & $18(7-36)$ \\
Urea (mmol/l) & $3.23 \pm 3.74$ & $2.7(1-43)$ \\
Hematocrit $(\%)$ & $33.83 \pm 3.77$ & $33.9(22.7-43.9)$ \\
Hemoglobin $(\mathrm{g} / \mathrm{l})$ & $110.33 \pm 13.64$ & $110(71.2-151)$ \\
Mean corpuscular hemoglobin $(\mathrm{pg})$ & $24.59 \pm 3.21$ & $25.1(16-30)$ \\
Mean corpuscular hemoglobin concentration $(\mathrm{g} / \mathrm{l})$ & $324.45 \pm 17.91$ & $327(255-359)$ \\
Platelet count $(x 109 / \mathrm{l})$ & $230.19 \pm 124.99$ & $248(16-555)$ \\
Prothrombin time $(\mathrm{sec})$ & $12.84 \pm 1.11$ & $12.7(11-19.9)$ \\
Fibrin degradation product $(\mu \mathrm{g} / \mathrm{ml})$ & $11.53 \pm 18.02$ & $6.51(1.28-96.25)$ \\
\hline
\end{tabular}

Table II. Drugs used in combination with with sirolimus in the present study.

\begin{tabular}{lcc}
\hline Drug & Category & Number of patients \\
\hline Sulfamethoxazole & 0 & 1 \\
Methylprednisolone & 1 & 16 \\
Digoxin & 0 & 7 \\
& 1 & 10 \\
Ursodeoxycholic & 0 & 16 \\
& 1 & 1 \\
Cimetidine & 0 & 16 \\
& 1 & 1 \\
Omeprazole & 0 & 16 \\
& 1 & 1 \\
Fluconazole & 0 & 12 \\
Cefoperazone/sulbactam & 1 & 5 \\
Voriconazole & 0 & 16 \\
& 1 & 1 \\
& 0 & 13 \\
& 1 & 1 \\
\hline
\end{tabular}

The numbers of patients treated with or without each drug are shown. Categories: 0, without drug; 1, with drug.

Modeling. All covariates were evaluated and only the following covariates exhibited a statistically significant effect on the PK parameters: Age, ALT levels and sex. These were included as significant covariates for $\mathrm{CL} / \mathrm{F}$, and duration of treatment with sirolimus was included as a significant covariate for $\mathrm{V} / \mathrm{F}$. The changes of the OFV are presented in Table III. The final covariate
Table III. Change of OFV of covariate analysis.

\begin{tabular}{lccc}
\hline A, Inclusion & & & \\
\hline & & $\begin{array}{c}\text { Changed } \\
\text { OFV }\end{array}$ & P-value \\
Model description & OFV & n/a & n/a \\
\hline Base model & 632.127 & -19.965 & $<0.05$ \\
Influence of DTT on V/F & 612.162 & -12.025 & $<0.05$ \\
Influence of age on CL/F & 600.137 & -5.69 & $<0.05$ \\
Influence of ALT on CL/F & 594.447 & -16.638 & $<0.05$ \\
Influence of sex on CL/F & 577.809 & & \\
\hline B, Elimination & & & \\
\hline & & & \\
& & & \\
Model description & OFV & OFV & P-value \\
\hline Full model & 577.809 & n/a & n/a \\
Eliminate DTT on V/F & 585.027 & 7.218 & $<0.01$ \\
Eliminate age on CL/F & 591.406 & 13.597 & $<0.01$ \\
Eliminate ALT on CL/F & 599.727 & 21.918 & $<0.01$ \\
Eliminate sex on CL/F & 594.447 & 16.638 & $<0.01$ \\
\hline
\end{tabular}

ALT, alanine transaminase; CL/F, apparent oral clearance (1/h); DTT, duration of treatment with sirolimus; $\mathrm{OFV}$, objective function values; $\mathrm{V} / \mathrm{F}$, apparent volume of distribution (1).

models were as follows: $\mathrm{CL} / \mathrm{F}=\theta_{\mathrm{CL} / \mathrm{F}} \mathrm{x} \operatorname{EXP}\left(\theta_{\mathrm{AGE}} \mathrm{x}\right.$ age $) \mathrm{x}$ $\operatorname{EXP}\left(\theta_{\mathrm{ALT}} \mathrm{x}\right.$ ALT $) \times \operatorname{EXP}\left(\theta_{\mathrm{SEX}} \times \operatorname{sex}\right) ; \mathrm{V} / \mathrm{F}=\theta_{\mathrm{V} / \mathrm{F}} \mathrm{x} \operatorname{EXP}\left[\theta_{\mathrm{DTT}} \mathrm{x}\right.$ (DTT/10)]. Where EXP is exponential function, $\theta_{\mathrm{CL} / \mathrm{F}}$ and $\theta_{\mathrm{V} / \mathrm{F}}$ are the typical population values of $\mathrm{CL} / \mathrm{F}$ and $\mathrm{V} / \mathrm{F}$, respectively. $\theta_{\mathrm{AGE}}$, $\theta_{\mathrm{ALT}}, \theta_{\mathrm{SEX}}$ and $\theta_{\mathrm{DTT}}$ are the coefficients of the age, ALT, sex and duration of treatment with sirolimus, respectively. 


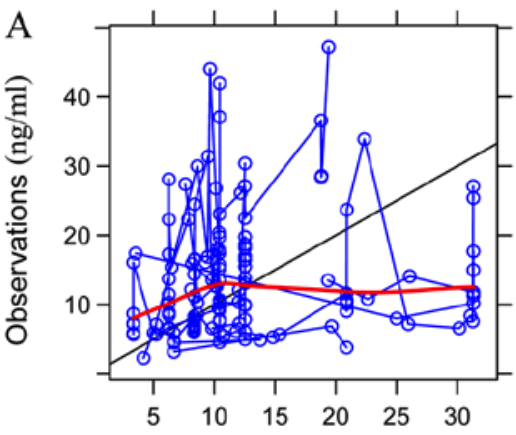

Population predictions (ng/ml)
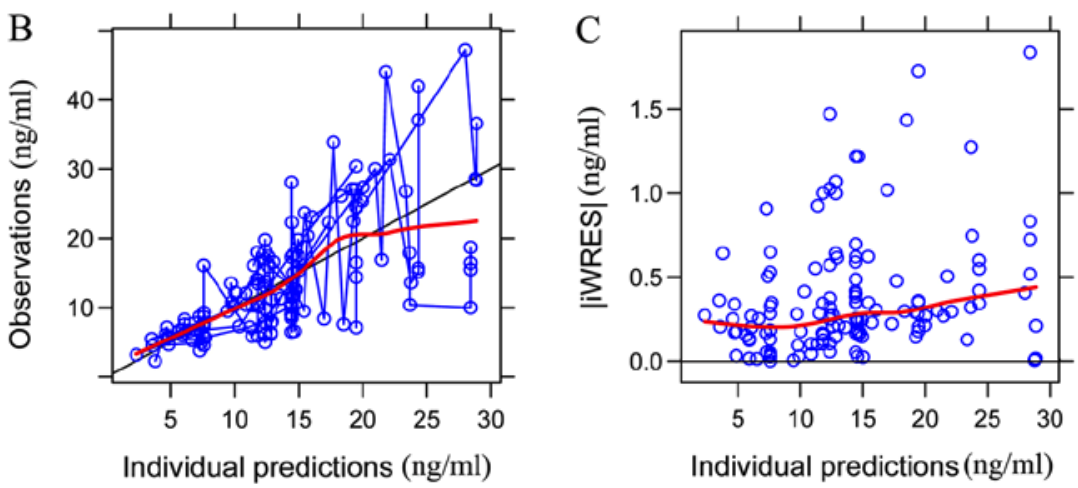

Figure 1. Goodness-of-fit plots of the base population model. (A) Observations vs. population predictions. The x-axis value of a point is the population prediction of sirolimus blood concentration and the $\mathrm{y}$-axis value of a point is the observation of sirolimus blood concentration. The closer the $\mathrm{x}$ - and $\mathrm{y}$-axis values of the same point are, namely, the closer the point is to the $y=x$ line, the closer the population prediction value predicted by the model is to the observation of sirolimus blood concentration. The black solid line is the line of unity (the $\mathrm{y}=\mathrm{x}$ line). The red smooth line represents the trend of the points. Hence, the closer the red smooth line is to the black solid line, the more predictive the model is. (B) Observations vs. individual predictions. The $\mathrm{x}$-axis value of a point is the individual prediction of sirolimus blood concentration and the $\mathrm{y}$-axis value of a point is the observation of sirolimus blood concentration. The closer the $\mathrm{x}$ - and $\mathrm{y}$-axis values of the same point are, namely, the closer the point is to the $\mathrm{y}=\mathrm{x}$ line, the closer the individual prediction value predicted by the model is to the observation of sirolimus blood concentration. The black solid line is the line of unity (the $y=x$ line). The red smooth line represents the trend of the points. Hence, the closer the red smooth line is to the black solid line, the more predictive the model is. (C) |iWRES| vs. individual predictions. iWRES is the difference in the values of the individual prediction and the observation of sirolimus blood concentration. The $\mathrm{x}$-axis value of a point is the individual prediction of sirolimus blood concentration and the y-axis value of a point is the iWRES of the corresponding individual prediction. The smaller the iWRES the better the model predictability. The red smooth line represents the trend of the points. Hence, the closer the red smooth line is to the black solid line (the $y=0$ line), the more predictive the model is. iWRES, individual weighted residuals.

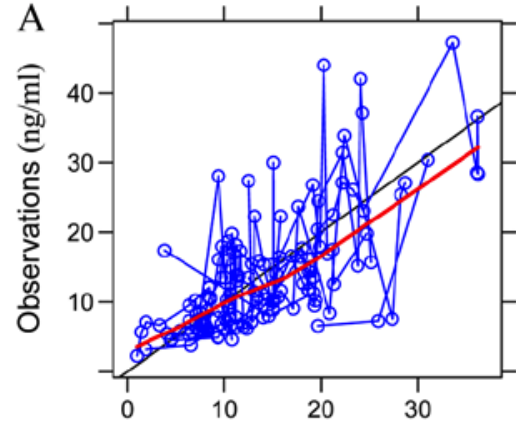

Population predictions $(\mathrm{ng} / \mathrm{ml})$
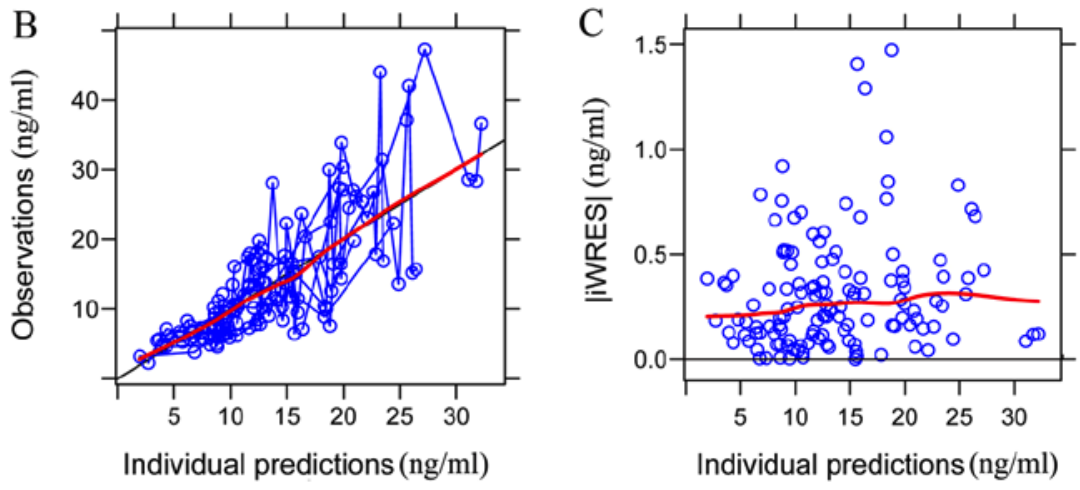

Figure 2. Goodness-of-fit plots of the final population model. (A) Observations vs. population predictions. The x-axis value of a point is the population prediction of sirolimus blood concentration and the $\mathrm{y}$-axis value of a point is the observed sirolimus blood concentration. The closer the $\mathrm{x}$ - and $\mathrm{y}$-axis values of the same point are, namely, the closer the point is to the $\mathrm{y}=\mathrm{x}$ line, the closer the population prediction value predicted by the model is to the observed sirolimus blood concentration. The black solid line is the line of unity (the $y=x$ line). The red smooth line represents the trend of the points. Therefore, the closer the red smooth line is to the black solid line, the more predictive the model is. (B) Observations vs. individual predictions. The $\mathrm{x}$-axis value of a point is the individual prediction of sirolimus blood concentration and the $\mathrm{y}$-axis value of a point is the observed sirolimus blood concentration. The closer the $\mathrm{x}$ - and $\mathrm{y}$-axis values of the same point are, namely, the closer the point is to the $\mathrm{y}=\mathrm{x}$ line, the closer the individual prediction value predicted by the model is to the observation of sirolimus blood concentration. The black solid line is the line of unity (the $y=x$ line). The red smooth line represents the trend of the points. Hence, the closer the red smooth line is to the black solid line, the more predictive the model is. (C) |iWRES| vs. individual predictions. iWRES is the difference in values between the individual prediction and the observed sirolimus blood concentration. The X-axis value of a point is the individual prediction of sirolimus blood concentration and the $y$-axis value of a point is the iWRES of the corresponding individual prediction. The smaller the iWRES value, the better the model predictability. The red smooth line represents the trend of the points. Therefore, the closer the red smooth line is to the black solid line (the $y=0$ line), the more predictive the model is. iWRES, individual weighted residuals.

Model evaluation. The goodness-of-fit plots of the base model (Fig. 1) were compared with those of the final model (Fig. 2). Black solid lines represent the line of unity and red smooth lines represent the trend of the data. The closer the red smooth line is to the black solid line, the more predictive the model is. The data in Fig. 2 exhibited an improved closeness degree compared with the data in Fig. 1, since Fig. 2 added covariates (age, ALT, sex and DTT) based on Fig. 1, demonstrating that the final model was better fitted to observed concentrations. Parameter estimates obtained with the final model and the bootstrap validation are presented in Table IV. The median values of the parameter estimate obtained via bootstrap validation were close to the respective values from the final population model, which demonstrated that the estimates for the PK parameters in the final population model were accurate, and that the model was reliable. The distribution of weighted residuals for the final model is presented in Fig. 3, which was approximately normally distributed. The prediction-corrected visual predictive check plot for the final model is presented in Fig. 4. The majority of 
Table IV. Parameter estimates of the final model and bootstrap validation.

\begin{tabular}{lccccc}
\hline & & & \multicolumn{2}{c}{ Bootstrap } \\
\cline { 4 - 5 } Parameter & Estimate & SE $(\%)$ & Median & $95 \% \mathrm{CI}$ & Bias (\%) \\
\hline $\mathrm{CL} / \mathrm{F}(\mathrm{l} / \mathrm{h})$ & 3.19 & 23.7 & 3.21 & {$[1.83,5.46]$} & 0.63 \\
$\mathrm{~V} / \mathrm{F}(\mathrm{liters})$ & 165 & 26.9 & 159 & {$[56.12,389.07]$} & -3.64 \\
$\mathrm{~K}_{\mathrm{a}}\left(\mathrm{h}^{-1}\right)$ & $0.485(\mathrm{fixed})$ & - & - & - & - \\
$\theta_{\mathrm{AGE}}$ & 0.215 & 21.7 & 0.21 & {$[0.09,0.35]$} & -4.65 \\
$\theta_{\mathrm{ALT}}$ & 0.0108 & 18 & 0.01 & {$[-0.01,0.014]$} & 2.78 \\
$\theta_{\mathrm{SEX}}$ & -0.818 & 15.4 & -0.8 & {$[-1.08,-0.52]$} & -2.51 \\
$\theta_{\mathrm{DTT}}$ & 0.0783 & 7.8 & 0.08 & {$[0.02,0.1]$} & 1.15 \\
$\omega_{\mathrm{CL} / \mathrm{F}}$ & 0.217 & 20.7 & 0.19 & {$[0.06,0.26]$} & -13.13 \\
$\omega_{\mathrm{VVIF}}$ & 1.334 & 15.2 & 1.19 & {$[0.67,1.86]$} & -11.02 \\
$\sigma_{1}$ & 0.365 & 6.8 & 0.36 & {$[0.31,0.4]$} & -1.78 \\
\hline
\end{tabular}

SE, standard error. The $95 \% \mathrm{CI}$ was presented as the 2.5 th and 97.5 th percentile of bootstrap estimates. Bias $=($ median-estimate)/estimate $\mathrm{x} 100 \% . \theta_{\mathrm{AGE}}$, coefficient of the age; $\theta_{\mathrm{ALT}}$, coefficient of the alanine transaminase; $\theta_{\mathrm{DTT}}$, coefficient of the duration of treatment with sirolimus; $\theta_{\mathrm{SEX}}$, coefficient of the sex; $\omega_{\mathrm{CL} / \mathrm{F}}$, inter-individual variability of $\mathrm{CL} / \mathrm{F} ; \omega_{\mathrm{V} / \mathrm{F}}$, inter-individual variability of $\mathrm{V} / \mathrm{F}$; $\sigma_{1}$, residual variability; Bias, prediction error; CI, confidence interval; $\mathrm{CL} / \mathrm{F}$, apparent oral clearance $(1 / \mathrm{h}) ; \mathrm{K}_{\mathrm{a}}$, absorption rate constant $\left(\mathrm{h}^{-1}\right)$; V/F, apparent volume of distribution (1).
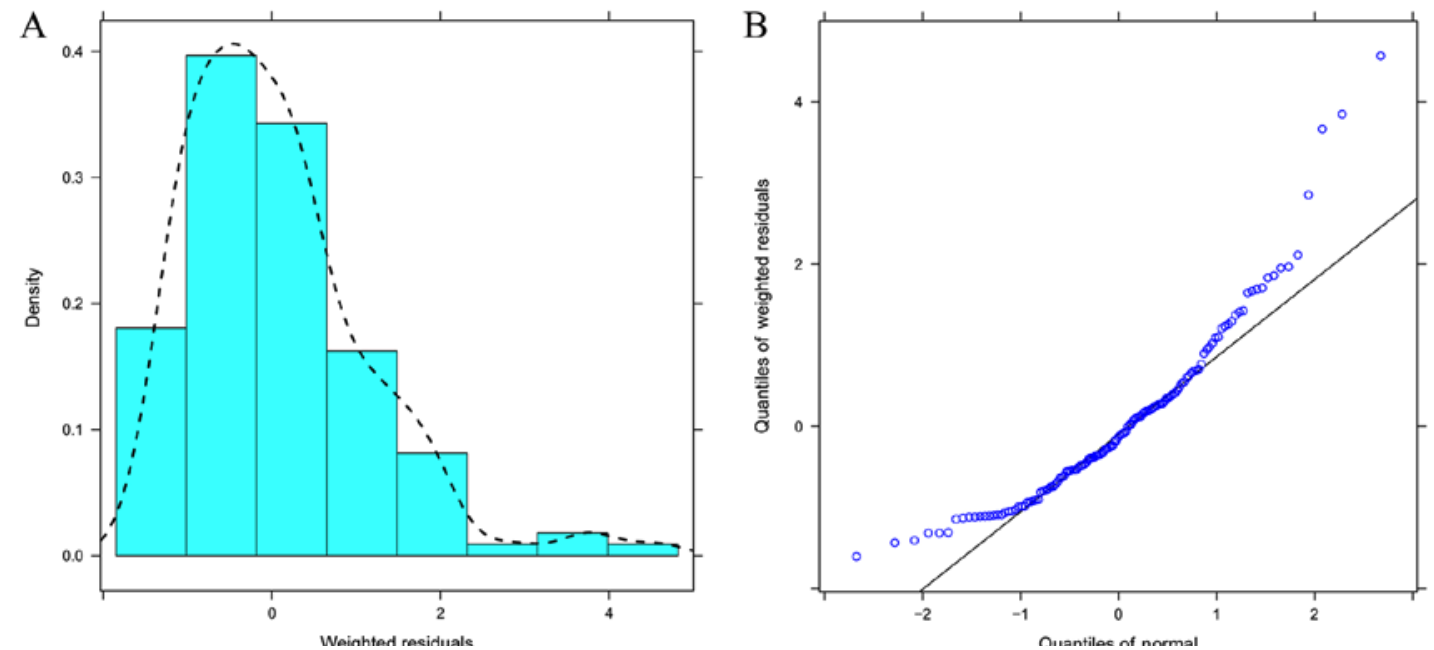

Figure 3. Distribution of weighted residuals for the final model. (A) Density vs. weighted residuals. The black dashed line is the trend line of weighted residuals distribution for the final model. The closer the trend line is to the normal distribution, the more stable the model is. (B) Quantiles of weighted residuals vs. quantiles of normal. The $\mathrm{x}$-axis value of a point is the quantiles of normal and the $\mathrm{y}$-axis value of a point is the quantiles of weighted residuals. The black solid line is the line of unity (the $y=x$ line). The closer the points are to the $y=x$ line, the more stable the model is.

the observed concentrations were within the $95 \%$ prediction intervals from the simulation data, indicating that the final model had an improved predictability.

\section{Discussion}

Sirolimus, an mTOR inhibitor, is expected to be effective in conditions associated with disorders of the mTOR growth control pathway (19). Sirolimus has been widely used in transplantation patients for the prevention of organ allograft rejection, and has been verified to be an effective treatment for PKHE (6-10).

The present study provided the first PPK analysis of sirolimus in Chinese patients with PKHE. Although the TDM data were not originally designed to investigate the PK characteristics of sirolimus, the population approach provides a powerful tool to extract useful information from sparse sampling data (20). Therefore, the present study may help to optimize the use of sirolimus by facilitating the achievement of the desirable therapeutic concentration. In addition, the model of the present study is ethically acceptable for studying pediatric patients, since no excessive blood sampling is required, while a larger amount of blood samples may have been required for traditional PK studies (21). In addition, the sirolimus PPK model in the present study has clinical value in predicting the PK in individual patients with PKHE.

Previously, a developed sirolimus maturation model $(22,23)$ was used to simulate clearance estimates using realistic age and weight covariates for age cohorts aged 0-24 months. In addition, 


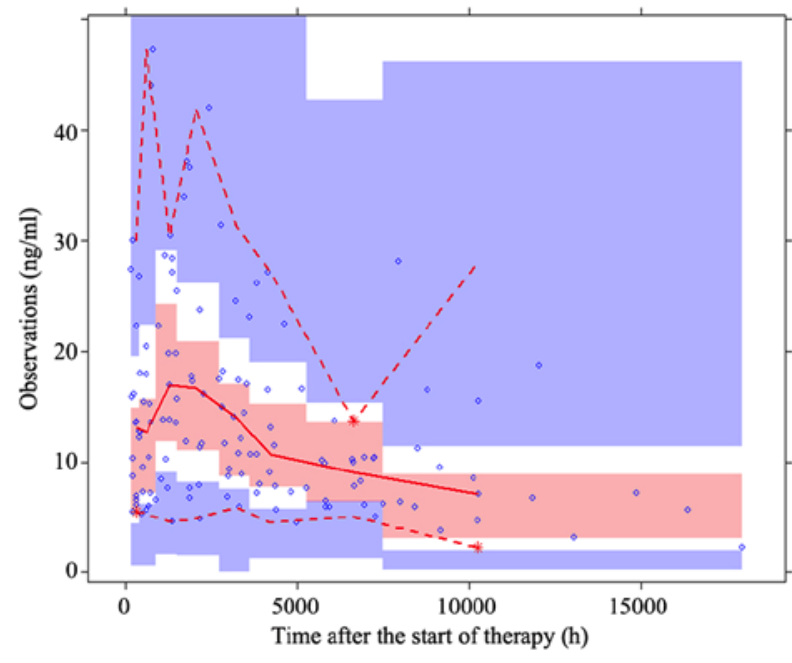

Figure 4. Prediction-corrected visual predictive check for the final model. The solid red line represents the median of the prediction-corrected concentrations of the final model. The lower and upper red dashed lines are the 2.5th and 97.5 th percentiles of the prediction-corrected concentrations of the final model, respectively, representing the lower and upper limits of the $95 \% \mathrm{CI}$ of predicted values. The blue points are observed concentrations (measured concentrations). Theoretically, the higher the number of the measured concentrations included in the $95 \%$ CI of predicted values, the better the predictability of the model. CI, confidence interval.

Mizuno et al (24) reported on model-based precision dosing of sirolimus in pediatric patients with vascular anomalies. The final model included a maturation function for sirolimus clearance and allometrically scaled body weight to account for size differences. However, their models were based on the precondition that the patients had adequate liver and renal functions and did not receive any cytochrome P450 family 3 subfamily A member 4 (CYP3A4) inhibitors or inducers. To explore the influence of demographic features, biological characteristics and concomitant medications on sirolimus CL/F in Chinese patients with PKHE, the present PPK model was built. A one-compartment model with first-order absorption was used to describe the absorption phase. Age, ALT and sex influenced the $\mathrm{CL} / \mathrm{F}$, while the duration of treatment with sirolimus influenced the V/F.

Sirolimus is mainly metabolized by the gut mucosa and liver $(25,26)$, and the liver is the major metabolism organ of sirolimus, whose function can affect the drug clearance. In the present study, ALT had an influence on the CL/F of sirolimus, which was similar to the observation regarding the $\mathrm{CL} / \mathrm{F}$ of tacrolimus reported by Yang et al (27). In addition, growth and development may be investigated using readily observable demographic factors, including weight and age (28). Weight of an individual was selected as a covariate, not body mass index, since Emoto et al (22) and Mizuno et al (23) also selected the weight of an individual as a covariate. Of note, referring to the present study, weight and age exhibited co-linearity and were positively associated. Furthermore, sex and weight also exhibited an association. Numerous studies have demonstrated that the association between drug clearance and body weight in pediatric patients is non-linear and may be well described using allometric scaling (29-31). Size is the primary covariate and may be referenced to a $70-\mathrm{kg}$ person by allometry using a coefficient of 0.75 for clearance (28). The allometric scaling model was assessed in the present study. The present study revealed that body weight is directly associated with sirolimus clearance, and that age and sex are indirect markers. However, the covariates age and sex in combination exhibited smaller OFV and they were, therefore, used in combination in the present study. The present study indicated that $\mathrm{CL} / \mathrm{F}$ increased with age, and that males had a higher $\mathrm{CL} / \mathrm{F}$ than females of the same age. This was expected, as infants/children gain weight as their age increases, and males have a higher body weight than females. The present results are essentially consistent with allometric scaling. However, the combination of age and sex is more suitable for modeling the trough concentration data obtained from traditional TDM. Similarly, previous studies have also indicated that patient sex $(32)$ and age $(33,34)$ affect the CL/F of sirolimus. Furthermore, the duration of treatment with sirolimus was significantly associated with the $\mathrm{V} / \mathrm{F}$ of sirolimus in the present study. One possible explanation is that sirolimus widely distributes in the erythrocytes. As the health of the patients with PKHE recovers, erythrocyte values increase after treatment (Table SI), and this may explain the present observation of the increased $\mathrm{V} / \mathrm{F}$ with increasing duration of treatment with sirolimus. In addition, the 17 patients with PKHE in the present study were co-treated with various other drugs: Sulfamethoxazole, methylprednisolone, digoxin, ursodeoxycholic, cimetidine, omeprazole, fluconazole, cefoperazone/sulbactam and voriconazole. However, no significant drug-drug interactions were identified in the analysis of the PK of sirolimus.

There were several limitations in the present study. First, since only the trough concentration data were available, only a one-compartment model could be applied due to the lack of sufficient informative data regarding drug absorption and distribution. It was not possible to estimate $\mathrm{F}$ and the absorption rate constant with a lag time. This is a common modeling method for sparse data processing. In Jiao et al (17), Yang et al (27) and Wang et al (15), it was also not possible to estimate F. Furthermore, Emoto et al (35) reported the impact of CYP3A5*3 polymorphism on sirolimus PK. However, currently, this pharmacogenomic consideration for sirolimus has not been verified in clinical studies. The present study was retrospectively performed using real-world data, which means that genotyping was not routinely performed in the population assessed in the present study. Pharmacogenomic data was also not included in the sirolimus PPK studies by Jiao et al (17) and Wang et al (36). Whether the inclusion of genotyping in the PPK model may better account for the variabilities of sirolimus in patients with PKHE should be investigated. In addition, the limited sample size was due to low incidence, and other studies have used similar group sizes (37). Furthermore, information regarding how large the PKHE lesion was in each patient with treatment time was an evaluation index of pharmacodynamics. Population pharmacokinetics and pharmacodynamics of sirolimus in pediatric patients with KHE will be further established in the future.

In conclusion, the first PPK model of sirolimus in Chinese patients with PKHE was established using retrospective data from a real-world population obtained by routine monitoring. The typical values of $\mathrm{CL} / \mathrm{F}$ and $\mathrm{V} / \mathrm{F}$ in the final model were $3.19 \mathrm{l} / \mathrm{h}$ and 165 liters, respectively. Age, ALT and sex were included as significant covariates for $\mathrm{CL} / \mathrm{F}$, and the duration of treatment with sirolimus was a significant covariate for $\mathrm{V} / \mathrm{F}$. 


\section{Acknowledgements}

Not applicable.

\section{Funding}

The present study was supported by the Clinical Pharmacy Key Specialty Construction Project of Shanghai (grant no. YZ2017/5), the Young Medical Talents of Wuxi (grant no. QNRC020), the Young Project of Wuxi Health and Family Planning Research (grant no. Q201706), the AOSAIKANG Pharmaceutical Foundation (grant no. A201826) and the Wuxi Science and Technology Development Guidance Plan (Medical and Health Care; grant no. CSZON1744).

\section{Availability of data and materials}

The datasets used and/or analyzed during the present study are available from the corresponding author on reasonable request.

\section{Authors' contributions}

ZL conceived and designed the study. DW and XC collected the data and built the model. DW wrote the paper. $\mathrm{XC}$ reviewed and edited the manuscript. All authors read and approved the final manuscript.

\section{Ethics approval and consent to participate}

The study was approved by the Research Ethics Committee of Children's Hospital of Fudan University without the need for written informed consent, since the data were retrospectively collected without patient identifiers.

\section{Patient consent for publication}

Not applicable.

\section{Competing interests}

The authors declare that they have no competing interests.

\section{References}

1. Reichel A, Hamm H, Wiegering V, Wiewrodt B, Neubauer H, Ernestus K and Winkler B: Kaposiform hemangioendothelioma with Kasabach-Merritt syndrome: Successful treatment with sirolimus. J Dtsch Dermatol Ges 15: 329-331, 2017.

2. DeFatta RJ, Verret DJ, Adelson RT, Gomez A and Myers LL: Kaposiform hemangioendothelioma: Case report and literature review. Laryngoscope 115: 1789-1792, 2005.

3. Yasui N, Koh K, Kato M, Park MJ, Tomizawa D, Oshima K, Uchisaka N,Gocho Y,Arakawa A,Seki M, et al: Kasabach-Merritt phenomenon: A report of 11 cases from a single institution. J Pediatr Hematol Oncol 35: 554-558, 2013.

4. Croteau SE, Liang MG, Kozakewich HP, Alomari AI, Fishman SJ, Mulliken JB and Trenor CC III: Kaposiform hemangioendothelioma: Atypical features and risks of Kasabach-Merritt phenomenon in 107 referrals. J Pediatr 162: 142-147, 2013.

5. Yadav D, Maheshwari A, Aneja S, Seth A and Chandra J: Neonatal Kasabach-Merritt phenomenon. Indian J Med Paediatr Oncol 32: 238-241, 2011.

6. Blatt J, Stavas J, Moats-Staats B, Woosley J and Morrell DS: Treatment of childhood kaposiform hemangioendothelioma with sirolimus. Pediatr Blood Cancer 55: 1396-1398, 2010.
7. Iacobas I, Simon ML, Amir T, Gribbin CE, McPartland TG, Kaufman MR, Calderwood S and Nosher JL: Decreased vascularization of retroperitoneal kaposiform hemangioendothelioma induced by treatment with sirolimus explains relief of symptoms. Clin Imaging 39: 529-532, 2015.

8. Jahnel J, Lackner H, Reiterer F, Urlesberger B and Urban C: Kaposiform hemangioendothelioma with Kasabach-Merritt phenomenon: From vincristine to sirolimus. Klin Padiatr 224: 395-397, 2012.

9. Kai L, Wang Z, Yao W, Dong K and Xiao X: Sirolimus, a promising treatment for refractory Kaposiform hemangioendothelioma. J Cancer Res Clin Oncol 140: 471-476, 2014.

10. Adams DM, Trenor CC III, Hammill AM, Vinks AA, Patel MN, Chaudry G, Wentzel MS, Mobberley-Schuman PS, Campbell LM, Brookbank C, et al: Efficacy and safety of sirolimus in the treatment of complicated vascular anomalies. Pediatrics 137: e20153257, 2016.

11. Zimmerman JJ and Kahan BD: Pharmacokinetics of sirolimus in stable renal transplant patients after multiple oral dose administration. J Clin Pharmacol 37: 405-415, 1997.

12. MacDonald A, Scarola J, Burke JT and Zimmerman JJ: Clinical pharmacokinetics and therapeutic drug monitoring of sirolimus. Clin Ther 22 (Suppl B): B101-B121, 2000.

13. Chevillard L, Sabo N, Tod M, Labat L, Chasport C, Chevaleyre C, Thibaut F, Barre J, Azuar J, Questel F, et al: Population pharmacokinetics of oral baclofen at steady-state in alcoholic-dependent adult patients. Fundam Clin Pharmacol 32: 239-248, 2018.

14. Marsot A, Michel F, Chasseloup E, Paut O, Guilhaumou R and Blin O: Phenobarbital in intensive care unit pediatric population: Predictive performances of population pharmacokinetic model. Fundam Clin Pharmacol 31: 558-566, 2017.

15. Wang DD, Lu JM, Li Q and Li ZP: Population pharmacokinetics of tacrolimus in paediatric systemic lupus erythematosus based on real-world study. J Clin Pharm Ther 43: 476-483, 2018.

16. Vadcharavivad S, Praisuwan S, Techawathanawanna N, Treyaprasert $\mathrm{W}$ and Avihingsanon Y: Population pharmacokinetics of tacrolimus in Thai kidney transplant patients: Comparison with similar data from other populations. J Clin Pharm Ther 41: 310-328, 2016.

17. Jiao Z, Shi XJ, Li ZD and Zhong MK: Population pharmacokinetics of sirolimus in de novo Chinese adult renal transplant patients. Br J Clin Pharmacol 68: 47-60, 2009.

18. Jonsson EN and Karlsson MO: Xpose-an S-PLUS based population pharmacokinetic/pharmacodynamic model building aid for NONMEM. Comput Methods Programs Biomed 58: 51-64, 1999.

19. Fasolo A and Sessa C: Current and future directions in mammalian target of rapamycin inhibitors development. Expert Opin Investig Drugs 20: 381-394, 2011.

20. Thomson AH and Whiting B: Bayesian parameter estimation and population pharmacokinetics. Clin Pharmacokinet 22: 447-467, 1992.

21. Kauffman RE and Kearns GL: Pharmacokinetic studies in paediatric patients. Clinical and ethical considerations. Clin Pharmacokinet 23: 10-29, 1992.

22. Emoto C, Fukuda T, Mizuno T, Schniedewind B, Christians U, Adams DM and Vinks AA: Characterizing the developmental trajectory of sirolimus clearance in neonates and infants. CPT Pharmacometrics Syst Pharmacol 5: 411-417, 2016.

23. Mizuno T, Fukuda T, Emoto C, Mobberley-Schuman PS, Hammill AM, Adams DM and Vinks AA: Developmental pharmacokinetics of sirolimus: Implications for precision dosing in neonates and infants with complicated vascular anomalies. Pediatr Blood Cancer: 64, 2017 doi: 10.1002/pbc.26470.

24. Mizuno T, Emoto C, Fukuda T, Hammill AM, Adams DM and Vinks AA: Model-based precision dosing of sirolimus in pediatric patients with vascular anomalies. Eur J Pharm Sci 109S: S124-S131, 2017.

25. Lampen A, Zhang Y, Hackbarth I, Benet LZ, Sewing KF and Christians U: Metabolism and transport of the macrolide immunosuppressant sirolimus in the small intestine. J Pharmacol Exp Ther 285: 1104-1112, 1998 .

26. Sattler M, Guengerich FP, Yun CH, Christians U and Sewing KF: Cytochrome P-450 3A enzymes are responsible for biotransformation of FK506 and rapamycin in man and rat. Drug Metab Dispos 20: 753-761, 1992.

27. Yang JW, Liao SS, Zhu LQ, Zhao Y, Zhang Y, Sun XY, Rao W, $\mathrm{Qu} \mathrm{W}, \mathrm{Li}$ WZ and Sun LY: Population pharmacokinetic analysis of tacrolimus early after Chinese pediatric liver transplantation. Int J Clin Pharmacol Ther 53: 75-83, 2015. 
28. Anderson BJ and Holford NH: Mechanism-based concepts of size and maturity in pharmacokinetics. Annu Rev Pharmacol Toxicol 48: 303-332, 2008

29. Wang DD, Chen X and Li ZP: Wuzhi capsule and haemoglobin influence tacrolimus elimination in paediatric kidney transplantation patients in a population pharmacokinetics analysis: A retrospective study. J Clin Pharm Ther: Mar 12, 2019 doi: 10.1111/jcpt.12828 (Epub ahead of print).

30. Wang D, Chen X, Xu H and Li Z: Population pharmacokinetics and dosing regimen optimisation of tacrolimus in Chinese pediatric hematopoietic stem cell transplantation patients. Xenobiotica: 1-8, 2019 (Epub ahead of print).

31. Wang D, Chen X and Li Z: Cyclosporin population pharmacokinetics in pediatric refractory nephrotic syndrome based on real-world studies: Effects of body weight and spirolactone administration. Exp Ther Med 17: 3015-3020, 2019.

32. Zimmerman JJ: Exposure-response relationships and drug interactions of sirolimus. AAPS J 6: e28, 2004.

33. Dansirikul C, Morris RG, Tett SE and Duffull SB: A Bayesian approach for population pharmacokinetic modelling of sirolimus. Br J Clin Pharmacol 62: 420-434, 2006.

34. Tejani A, Alexander S, Ettenger R, Lerner G, Zimmerman J, Kohaut E and Briscoe DM: Safety and pharmacokinetics of ascending single doses of sirolimus (Rapamune, rapamycin) in pediatric patients with stable chronic renal failure undergoing dialysis. Pediatr Transplant 8: 151-160, 2004.
35. Emoto C, Fukuda T, Venkatasubramanian R and Vinks AA: The impact of CYP3A5*3 polymorphism on sirolimus pharmacokinetics: Insights from predictions with a physiologically-based pharmacokinetic model. Br J Clin Pharmacol 80: 1438-1446, 2015.

36. Wang M, Duan BL, Yuan YJ, Su X, Zheng H, Wang FS and Sun H: Population pharmacokinetic characteristics of sirolimus in healthy Chinese subjects and renal transplant patients. Int J Clin Pharmacol Ther 54: 433-441, 2016.

37. Mizuno T, Fukuda T, Christians U, Perentesis JP, Fouladi M and Vinks AA: Population pharmacokinetics of temsirolimus and sirolimus in children with recurrent solid tumours: A report from the Children's Oncology Group. Br J Clin Pharmacol 83: 1097-1107, 2017.

(i) $($ ) This work is licensed under a Creative Common Attribution-NonCommercial-NoDerivatives 4.0 International (CC BY-NC-ND 4.0) License. 